\title{
Dependence of IGS Products on the ITRF Datum
}

\author{
J.R. Ray \\ NOAA National Geodetic Survey, 1315 East-West Highway, Silver Spring, Maryland 20910, USA \\ P. Rebischung \\ Institut Géographique National / LAREG, 6-8 Av. Blaise Pascal, 77455 Marne-la-Vallée, France \\ R. Schmid \\ Institut für Astronomische und Physikalische Geodäsie, Technische Universität München, Germany
}

\begin{abstract}
Throughout its nearly two decades, the International GNSS (Global Navigation Satellite Systems) Service (IGS) has sought to align its products closely to successive realizations of the International Terrestrial Reference Frame (ITRF). This has been disruptive for IGS users at times, especially during the 1990s when some radical ITRF datum choices were adopted. During the past decade, IGS impacts due to ITRF updates have been smaller and mostly caused by errors in the results from the contributing space geodetic techniques.
\end{abstract}

Frame orientations (rotations) are purely conventional, so the IGS relies on the ITRF via a subset of reliable, globally distributed stations. Except for the period when ITRF93 was used, this procedure has worked well. The IGS origin in principle could be self-reliant or contributory to ITRF by direct observation of a frame origin aligned to the long-term center of mass of the entire Earth system. In practice, however, GNSS-based results have been less reliable than those from satellite laser ranging (SLR). So the ITRF origin, based on SLR only, has been adopted historically. Until the transition from ITRF2005 to ITRF2008, there have sometimes been significant origin shifts as SLR results have evolved. However, the present stability of the ITRF origin may finally have reached the few-mm level.

In many respects, the IGS dependence on the ITRF scale is most subtle and problematic. In addition to an overall Helmert alignment of the IGS frame to match the ITRF scale (and other datum parameters), since 2006 the IGS calibration values for the GNSS satellite antenna $z$-offsets depend directly on the same ITRF scale (due to high correlations if the IGS frame scale is not fixed). We therefore face a non-linear situation to maintain full consistency between all IGS products and the ITRF scale: each IGS frame contribution to ITRF based on one set of antenna calibrations must be used, together with frames from other techniques, to determine an updated ITRF and new antenna calibrations, which are then no longer strictly consistent with the starting IGS frame. One can hope that the process will iteratively converge eventually. But large shifts in the ITRF scale, such as the -1 ppb change from ITRF2005 to ITRF2008, are highly disturbing, much more so than the associated rotational or translational shifts.

Only SLR and very long baseline interferometry (VLBI) have been considered reliable and accurate enough to be used for the ITRF scale. But experience and theoretical studies have shown that neither is accurate to better than about $1 \mathrm{ppb}$. Note in particular that a $1 \mathrm{ppb}$ uncertainty in the GM constant fundamentally limits the possible scale agreement between SLR and VLBI to no better. Consequently, the authors strongly urge that the ITRF scale hereafter be fixed conventionally to the ITRF2008 scale indefinitely until it is convincingly shown that VLBI and/or SLR can determine the ITRF scale within $0.5 \mathrm{ppb}$. If this is not done, the IGS might maintain its own ITRF2008 scaled frame to minimize future operational dislocations.

Keywords. Reference frames, International GNSS Service (IGS), International Terrestrial Reference Frame (ITRF), terrestrial scale, antenna calibration

\section{Introduction}

Over its 17-year history, the IGS has tried to adopt the references of the International Earth Rotation and Reference Systems Service (IERS) as closely as possible. The IERS Conventions are largely implemented, UT1 reference values are taken from the IERS (but propagated to data epochs via integration of IGS length-of-day observations), and successive ITRFxx datums have been used for IGS products. Starting in 2000, however, IGS internal frame realizations have been preferred in order to maintain the highest level of self-consistency, but aligned to the ITRF datum. 
Some difficulties have been encountered. The IERS Earth orientation parameters (EOPs) were found to be too inaccurate for direct use, so the IGS adopted its own observed pole starting in 1995. In addition, the datum shifts between ITRFxx updates have sometimes been disruptive for users. The large rotations applied to ITRF93 to reduce EOP inconsistencies were a particularly serious problem. Lately, the leading problem for the IGS has been change in the ITRF scale, because this affects estimates of GNSS satellite antenna z-offsets.

\section{ITRF Rotations}

Conventionally, each ITRF realization has been rotationally aligned to its predecessor, except for ITRF93, which was offset to restore consistency with IERS published EOPs. The rotations applied to ITRF93, compared to ITRF2008 at epoch 2000.0, were $-1.71,-1.48$, and -0.30 mas about $X, Y$, and $Z$, respectively (see Table 4.1 of Petit and Luzum, 2010). The rotation rates were non-zero also. After ITRF93, rotational consistency was restored.

The ITRF93 frame was used by the IGS from 1 Jan. 1995 (GPS week 782) till 29 Jun. 1996 (week 859). The original IGS Final orbits from that period were later compared to the homogeneously reprocessed orbits (IGS ACC, 2010) that used the IGS05 frame (aligned to ITRF2005). Gendt et al. (2010) found that large rotational offsets, up to nearly 1 mas, were evident in the ITRF93 orbits, closely matching the expected shifts due to the ITRF rotations. Figure 1 (from Gendt et al., 2010) illustrates results for the $X$ axis.

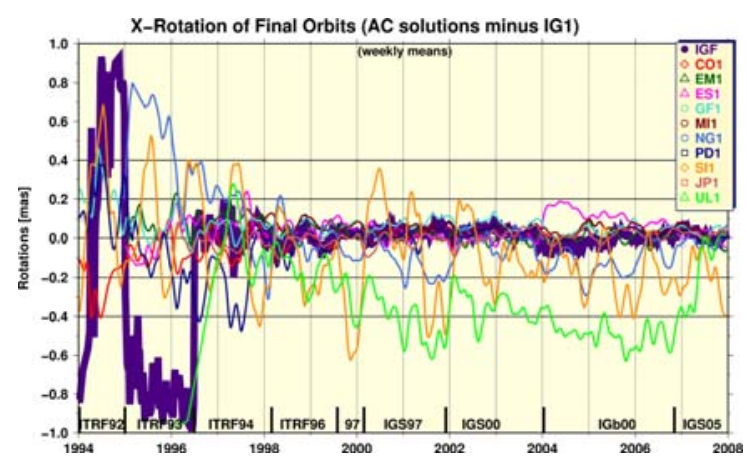

Fig. 1 The heavy purple line shows the $X$ rotations of the original IGS Final orbits compared to consistently reprocessed orbits. Frames used by the IGS are marked along the bottom of the plot, which comes from Gendt et al. (2010).

\section{ITRF Translations}

Since the first use of ITRF by the IGS (ITRF92 in 1994) the origin of successive frame realizations has moved by up to $-24.0,+4.6$, and $-41.2 \mathrm{~mm}$ in $X$, $Y$, and $Z$, respectively, compared to ITRF2008 at epoch 2000.0 (Petit and Luzum, 2010). The translation rates have reached up to $-3.2 \mathrm{~mm} / \mathrm{yr}$ in $Z$. From ITRF88 onward, shifts in the $Z$ direction have increased steadily from $-125.2 \mathrm{~mm}$ to the latest change of $-4.7 \mathrm{~mm}$ from ITRF2005 to ITRF2008. These are entirely due to the evolution of SLR geocenter results since no other technique contributes to the ITRF origin.

Contrary to the situation with ITRF rotations, the origin stability of the IGS orbit frame is not sensitive to the terrestrial frame origin. The results presented by Gendt et al. (2010) demonstrate this. The explanation is that the origin of IGS orbits should follow the actual Earth geocenter in the same way as for SLR, provided that no overconstraints are applied in the data analysis. However, the fidelity of IGS geocenter offsets is less reliable from GNSS measurements due to the greater importance of empirical orbit parameters. The original IGS orbit modeling caused a large drift in the $Y$ direction of more than $50 \mathrm{~mm}$ from 1994 till 1998, reflecting refined IGS orbit modeling as more once-per-revolution satellite parameters were added later. Orbit differences in the $X$ direction were minor but noisier before 1999 , whereas there was a strong annual variation in the $Z$ direction with an amplitude of $>10 \mathrm{~mm}$ before 2002, decreasing since then. The strong dependence of GPS geocenter $Z$ shifts on orbit modeling has been illustrated by Hugentobler (2005), who also found signals with periods equal to the GPS draconitic year (about 350 days). Such orbit-related effects dominate the IGS results over any impacts due to the ITRF origin.

\section{ITRF Scale and Satellite Antenna Offsets}

In 2006, the IGS adopted satellite antenna phase center offsets (PCOs, together with matching nadirdependent phase center variations, PCVs) that were determined from the GNSS data to be consistent with "absolute" calibrations for the ground tracking antennas (Schmid et al., 2007). Even using long spans of tracking data, the PCO estimation problem is not robust because the data are only weakly sensitive to PCO errors (Cardellach et al., 2007): 


$$
\Delta \rho=-\Delta \mathrm{PCO} *\left(0.94+0.06 \sin ^{2} e\right)^{1 / 2}
$$

where $\Delta \rho$ is the geometric range change between a satellite and a ground station due to a PCO error, $\triangle \mathrm{PCO}$, in the radial $(\mathrm{z})$ direction toward the Earth and $e$ is the elevation angle of the satellite viewed from the station. In addition, the $\triangle \mathrm{PCO}$ parameters are highly correlated with station height and station zenith troposphere delay parameters, whose partial derivatives are proportional to $\sin (e)$ and $1 / \sin (e)$, respectively. In order to solve this system sensibly, the IGS has chosen to fix the terrestrial frame scale (i.e., the net height of a global set of stations) to the ITRF scale (determined by VLBI and SLR data) and use absolute robot calibrations for the tracking antennas. Doing this, the expected error (in $\mathrm{mm}$ ) in $\triangle \mathrm{PCO}$ estimates averaged over the satellite constellation is about $-\left(20^{*} \Delta s\right)$ where $\Delta s$ is the net scale frame error (in $\mathrm{mm}$ ).

Since 1994, ITRFxx scales have differed from ITRF2008 by up to $3.41 \mathrm{ppb}$ or $21.7 \mathrm{~mm}$. The scale accuracy of the present ITRF2008 is thought to be about $1.2 \mathrm{ppb}$ or $8 \mathrm{~mm}$ (Altamimi et al., 2011), implying a frame-related error in IGS estimates of satellite PCOs of about $16 \mathrm{~cm}$. Because there are no near-term prospects for much better VLBI or SLR scale accuracies (noting the estimated error in $\mathrm{GM}$ alone, which scales all satellite frames, is about $1 \mathrm{ppb}$; see Petit and Luzum, 2010), we strongly recommend that future ITRF realizations use scales fixed to ITRF2008 as a conventional standard. This practice would greatly simplify ITRF usage and eliminate needless variations in applications that have no basis in physical reality.

\section{Fixing ITRF Scale Would Simplify PCO Maintenance}

The present IGS methodology to maintain and update satellite antenna PCOs is an iterative process. A particular GNSS-based frame (IGSxx, closely aligned to a given ITRFxx) is adopted for an extended operational period and for occasional reprocessing of the historic raw data set. Accompanying the frame must be a set of absolute antenna calibrations for ground and satellite antennas, igsxx.atx in the ANTEX format, wherein the satellite PCOs need to be consistent with the IGSxx frame scale. Normally, major changes in IGSxx and igsxx.atx are not allowed during their periods of usage in order to avoid instabilities in user results. The long-term solutions resulting from this system are the IGS inputs to the next ITRFyy realization.
If the ITRFyy scale differs from its predecessor, it is then necessary to generate updated PCOs and a new set of igsyy.atx calibrations, adding at the same time any major calibration changes for existing ground antennas. Computing the new PCOs can be done by defining a new IGSyy frame derived from ITRFyy and adopting its datum. Then, prior IGS solution files are back-solved to estimate consistent PCOs by fixing the IGSyy scale. If the ITRF scale were fixed conventionally, at least for an extended period, then the PCO maintenance could be greatly simplified and any adjustments that might be needed would be much smaller. However, if calibration values for existing ground antennas are changed (e.g., due to more recent measurements) then it is still necessary to compute station position corrections for any stations affected by the antenna calibration changes.

This process should converge if major calibration changes for existing antennas decline, as expected, but only if the ITRF scale also becomes much more stable over time. Within the current accuracy of VLBI and SLR, this is most easily assured by fixing the ITRF scale conventionally.

\section{Competing Strategies for ITRF2008 Combination}

In preparing ITRF2008, independent combination solutions were formed by Institut Géographique National (IGN) and by Deutsches Geodätisches Forschungsinstitut (DGFI). The same technique inputs were used by both groups, but their internal strategies differed in ways that have been difficult to compare independently. One of the few objective ways to evaluate the overall performance of the two approaches is to compare their combined polar motion (PM) estimates to independent excitation measures from atmosphere and ocean angular momentum (AAM and OAM, respectively). Such comparisons of geodetic and geophysical excitations have been made for many years relying on the products of general circulation models for the atmosphere and the ocean, mostly following the development by Barnes et al. (1983).

Kouba (2010) has kindly performed such a comparison between test IGN and DGFI PM time series and AAM + OAM excitations. AAM values, four times daily, are from the NCEP reanalysis model (Salstein et al., 1997) where the inverted barometer assumption has been applied. Daily averaged values are formed around noon epochs to match the respective geodetic series epochs. OAM values come from the ECCO_kf080 model (Gross et al., 2005). Both series are from the IERS Global 
Geophysical Fluids Center at www.iers.org. The $\mathrm{PM}$ and (AAM + OAM) time series were compared over the period 27 Feb. 1997 to 26 Dec. 2008 using methods described by Kouba (2005). Crosscorrelations between the series are shown in Table 1 and residuals in Table 2. Also included is the IGS reprocessed PM time series, since this contribution dominates the combinations. The most recent 4.5year period was also considered by Kouba but the conclusions are unchanged.

Kouba's results show that the DGFI combination has the lowest correlations and highest residuals over all spans compared to the geophysical excitations. Only the differences over spans shorter than 30 days are significant at the $95 \%$ confidence level but the margin grows steadily for shorter intervals. The IGN and IGS series are nearly indistinguishable and always agree with (AAM + OAM) better than the DGFI PM. These independent comparisons strongly suggest that the DGFI procedures have introduced some measure of high-frequency noise into the combination, relative to the IGS and IGN combination, although the exact mechanism for that cannot be identified from these results alone.

Table 1. PM excitation $\left(\mathrm{Chi}_{1}\right.$ related to variations in the $y$ component and $\mathrm{Chi}_{2}$ in the $x$ component) correlations between IGS (reprocessed), IGN, and DFGI time series and AAM+OAM. Correlations are computed over the interval from 27 Feb. 1997 to 26 Dec. 2008. Differences of $\sim 0.006$ are significant at the $95 \%$ level. The largest correlations for each sliding window span are shown in red. Results are from Kouba (2010).

\begin{tabular}{ccccccc}
\hline \multirow{2}{*}{ Spans } & \multicolumn{3}{c}{ Chi $_{2}$} & & Chi $_{1}$ \\
\cline { 2 - 7 } & IGS & IGN & DGFI & IGS & IGN & DGFI \\
\hline all & 0.904 & 0.904 & 0.902 & 0.769 & 0.769 & 0.765 \\
\hline 30 d & 0.892 & 0.892 & 0.888 & 0.858 & 0.858 & 0.852 \\
\hline 5 d & 0.785 & 0.785 & 0.775 & 0.732 & 0.732 & 0.719 \\
\hline 3 d & 0.703 & 0.700 & 0.687 & 0.634 & 0.634 & 0.616 \\
\hline
\end{tabular}

Table 2. RMS of residuals (units are mas/d) between PM and AAM+OAM excitations for IGS (reprocessed), IGN, and DFGI time series computed over three different spans. The smallest residuals for each sliding window span are shown in red. Results are from Kouba (2010).

\begin{tabular}{ccccccc}
\hline \multirow{2}{*}{ Spans } & \multicolumn{3}{c}{ Chi $_{2}$} & & Chi $_{1}$ \\
\cline { 2 - 7 } & IGS & IGN & DGFI & IGS & IGN & DGFI \\
\hline all & 0.270 & 0.270 & 0.273 & 0.255 & 0.254 & 0.257 \\
\hline$<6 \mathrm{~d}$ & 0.162 & 0.162 & 0.173 & 0.139 & 0.139 & 0.148 \\
\hline$<3 \mathrm{~d}$ & 0.111 & 0.111 & 0.122 & 0.106 & 0.106 & 0.112 \\
\hline
\end{tabular}

\section{Conclusions}

The stability of the ITRF datum is critical for the general usefulness and continuity of the IGS products, especially the orientation and scale components. To improve from the present level of stability, considering the intrinsic scale uncertainty of $\sim 1 \mathrm{ppb}$, the authors urge that future ITRF realizations maintain the ITRF2008 scale as a convention. There is no benefit to users to experience scale jumps with each ITRF update within that range (i.e., height changes within about $\pm 6 \mathrm{~mm}$ ). The most direct impact on the IGS is for its satellite antenna $z$-offset estimates, which rely on constraining the terrestrial scale to a specific datum. Meanwhile, the geodetic observing techniques need to focus their research efforts toward improving the stability and accuracy of their products, including the datum aspects.

It would also benefit the IGS if IERS procedures for handling future ITRF updates were improved. Clear and respected schedules for each new realization should be agreed and the criteria used to evaluate test combinations should be well defined and objective to ensure the highest possible quality. Considering the various factors, including the time needed for the next grand reprocessing, the IGS suggests that the next ITRF realization after 2008 aims for a delivery in about 2013.

\section{Acknowledgements}

The private and very helpful contributions of Jan Kouba are greatly appreciated. Xavier Collilieux has been instrumental in developing the framework to determine PCO estimates using prior analysis solutions with a fixed terrestrial scale. All components of the IGS (Dow et al., 2009) have been indispensible in carrying out this work.

\section{References}

Altamimi, Z., X. Collilieux, L. Metivier (2011) ITRF2008: An improved solution of the International Terrestrial Reference Frame. $J$. Geod., doi: 10.1007/s00190-011-0444-4, in press.

Barnes, R.T.H., R. Hide, A.A. White, and C.A. Wilson (1983). Atmospheric angular momentum fluctuations, length-of-day changes and polar motion. Proc. R. Soc. Lond., doi : 10.1098/ rspa.1983.0050, A 387, pp. 31-73.

Cardellach, E., P. Elósegui, and J.L. Davis (2007). Global distortion of GPS networks associated 
with satellite antenna model errors. J. Geophys. Res., doi : 10.1029/2006JB004675, 112, B07405.

Dow, J.M., R.E. Neilan, and C. Rizos (2009). The International GNSS Service in a changing landscape of Global Navigation Satellite Systems. J. Geod., doi: 10.1007/s00190-0080300-3, 83(3-4), pp. 191-198.

Gendt, G., J. Griffiths, T. Nischan, and J. Ray (2010). IGS reprocessing - Summary of orbit/clock combination and first quality assessment. Presentation at IGS Workshop, Newcastle upon Tyne, UK, available at acc.igs.org/repro1/repro1_IGSW10.pdf.

Gross R.S., I. Fukumori, and D. Menemenlis (2005). Atmospheric and oceanic excitation of decadal-scale Earth orientation variations, $J$. Geophys. Res., doi: 10.1029/2004JB003565, 110, B09405.

Hugentobler, U. (2005). Models in GNSS data analysis. Presentation at COMET - Advances in GPS Data Processing and Modeling for Geodynamics, University College London.

IGS ACC (2010). IGS Analysis Center Coordinator. Website at acc.igs.org.

Kouba, J. (2005). Comparison of polar motion with oceanic and atmospheric angular momentum time series for 2-day to Chandler periods. $J$. Geod., doi: 10.1007/s00190-005-0440-7, 79(13), pp. 33-42.

Kouba, J. (2010). ITRF2008 and IGS repro1 polar motion comparisons with AAM+OAM. Private communication, available electronically at acc.igs.org/trf/ITRF08ERPcomp.pdf.

Petit, G., and B. Luzum, eds. (2010). IERS Conventions (2010). IERS Technical Note 36, Verlag des Bundesamts für Kartographie und Geodäsie, Frankfurt am Main, 179 pp.

Salstein D.A. and R.D. Rosen (1997). Global momentum and energy signals from reanalysis systems, in 7th Conf. on Climate Variations, American Meteorological Society, Boston, MA, pp. 344-348.

Schmid, R., P. Steigenberger, G. Gendt, M. Ge, and M. Rothacher (2007). Generation of a consistent absolute phase center correction model for GPS receiver and satellite antennas. J. Geod., doi: 10.1007/s00190-007-0148-y, 81(12), pp. 781798. 